\title{
A Theory of Entanglement
}

\author{
Stan Gudder \\ Department of Mathematics, University of Denver, Denver, Colorado, USA. E-mail:sgudder@du.edu \\ Editors: James F. Glazebrook \& Danko D. Georgiev \\ Article history: Submitted on July 11, 2020; Accepted on July 22, 2020; Published on July 27, 2020.
}

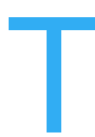
his article presents the basis of a theory of entanglement. We begin with a classical theory of entangled discrete measures. Then, we treat quantum mechanics and discuss the statistics of bounded operators on a Hilbert space in terms of context coefficients. Finally, we combine both topics to develop a general theory of entanglement for quantum states. A measure of entanglement called the entanglement number is introduced. Although this number is related to entanglement robustness, its motivation is not the same and there are some differences. The present article only involves bipartite systems and we leave the study of multipartite systems for later work.

Quanta 2020; 9: 7-15.

\section{Entangled Probability Measures}

Entangled states are considered to be an important resource for quantum computation and information processes [1-5]. Various authors have developed theories of entanglement [6-9] and this article is another attempt. Our motivation is a bit different and we hope this work will be useful.

It is frequently stated that entanglement is a strictly quantum phenomenon and it is not present in a classical theory. We do not believe this is actually true and begin with a classical theory of entangled measures. This theory is quite simple and does not have the depth of its quantum

(c) This is an open access article distributed under the terms of the Creative Commons Attribution License CC-BY-3.0, which permits unrestricted use, distribution, and reproduction in any medium, provided the original author and source are credited. counterpart. However, we believe that it can be instructive and give insights into the quantum theory.

Classical statistical systems are described by probability measures in a measure space. For simplicity we consider the set of probability measures $M$ on the set of natural numbers $\mathbb{N}$. We consider $\underline{u} \in M$ as a probability vector $\underline{u}=\left\{u_{i}: i \in \mathbb{N}\right\}, u_{i} \geq 0, \sum u_{i}=1$. Thinking of $M$ as a subset of the real Hilbert space

$$
\ell_{2}=\left\{f: \mathbb{N} \rightarrow \mathbb{R}, \sum|f(i)|^{2}<\infty\right\}
$$

we write $\|\underline{u}\|^{2}=\sum u_{i}^{2}$ and $\langle\underline{u}, \underline{v}\rangle=\sum u_{i} v_{i}$. The support of $\underline{u}$ is defined by

$$
\operatorname{supp}(\underline{u})=\left\{i \in \mathbb{N}: u_{i} \neq 0\right\} .
$$

The entanglement index of $\underline{u}$ is the cardinality of $\operatorname{supp}(\underline{u})$ and is denoted by $n(\underline{u})$. We define the entanglement number of $\underline{u}$ by $e(\underline{u})=\left(1-\|\underline{u}\|^{2}\right)^{1 / 2}$. We can also write

$$
e(\underline{u})=\left(\sum_{i \neq j} u_{i} u_{j}\right)^{1 / 2}=\left[\sum_{i} u_{i}\left(1-u_{i}\right)\right]^{1 / 2} .
$$

Notice that $\|\underline{u}\|^{2}$ is the expectation of the random variable $u_{i}$ relative to the measure $\underline{u}$ and that by $(1), e(\underline{u})^{2}$ is the expectation of the random variable $1-u_{i}$. That is, $e(\underline{u})^{2}$ is the average distance of $\underline{u}$ from unity. This is our first (among many) interpretations of $e(\underline{u})$. We say that $\underline{u}$ is a point (or Dirac) measure if $u_{i}=1$ for some $i \in \mathbb{N}$. Of course, it follows that $u_{j}=0$ for $j \neq i$. We say that $\underline{u}$ is uniform if $u_{i}=u_{j}$ whenever $u_{i}, u_{j} \neq 0$. If $\underline{u}$ is uniform, then $n(\underline{u})<\infty$ and $u_{i}=1 / n(\underline{u})$ whenever $u_{i} \neq 0$. The proof of the following result is standard. 
Theorem 1. (a) $e(\underline{u})=0$ if and only if $\underline{u}$ is a point measure. (b) If $n(\underline{u})<\infty$, then $e(\underline{u}) \leq[(n(\underline{u})-1) / n(\underline{u})]^{1 / 2}$ and equality is achieved if and only if $\underline{u}$ is uniform.

If $\underline{u}$ is uniform and $n(\underline{u}) \neq 1$ (equivalently $e(\underline{u}) \neq 0$ ), we say that $\underline{u}$ is maximally entangled with index $n(\underline{u})$. We conclude that there is precisely one maximally entangled probability measure for each nonsingleton finite support in $\mathbb{N}$. Moreover, $\underline{u}$ is maximally entangled if and only if $n(\underline{u}) \neq 1$ and $\|\underline{u}\|^{2}=1 / n(\underline{u})$. Of course, in this case $\underline{u}$ has the largest entanglement number of any $\underline{v} \in M$ with $n(\underline{v})=n(\underline{u})$. We also see that $0 \leq e(\underline{u})<1$ and since $\|\underline{u}\|>0$, there is no $\underline{u} \in M$ with $e(\underline{u})=1$.

Example 1. (a) If $u_{1}=u_{2}=1 / 2$, then $e(\underline{u})=1 / \sqrt{2}$ and $\underline{u}$ is maximally entangled with index 2. (b) If $u_{1}=$ $u_{2}=u_{3}=1 / 3$, then $e(\underline{u})=\sqrt{2 / 3}$ and $\underline{u}$ is maximally entangled with index 3 so the entanglement is larger than in (a). (c) If $u_{1}=1 / 2, u_{2}=1 / 3, u_{3}=1 / 6$, then $e(\underline{u})=$ $\sqrt{11 / 18}$ and

$$
\sqrt{\frac{1}{2}}<\sqrt{\frac{11}{18}}<\sqrt{\frac{2}{3}}
$$

(d) If $u_{1}=1 / 9, u_{2}=1 / 9, u_{3}=7 / 9$, then $e(\underline{u})=$ $\sqrt{30} / 9<1 / \sqrt{2}$. This gives the smallest entanglement of the four.
If $\underline{u}, \underline{v} \in M$ and $\lambda \in[0,1]$, then $\lambda \underline{u}+(1-\lambda) \underline{v} \in M$ is called a mixture of $\underline{u}$ and $\underline{v}$. It is easy to check that

$$
n[\lambda \underline{u}+(1-\lambda) \underline{v}]=n(\underline{u})+n(\underline{v})
$$

when $\lambda \in(0,1), \operatorname{supp}(\underline{u}) \cap \operatorname{supp}(\underline{v})=\emptyset$ and

$$
n[\lambda \underline{u}+(1-\lambda) \underline{v}] \leq n(\underline{u})+n(\underline{v})
$$

in general. However, we have that

$$
n[\lambda \underline{u}+(1-\lambda) \underline{v}] \geq \lambda n(\underline{u})+(1-\lambda) n(\underline{v}) .
$$

This last inequality says that the function $n$ is concave. We interpret this as saying that mixtures increase the entanglement index. We now show that the entanglement number is concave.

Theorem 2. For all $\underline{u}, \underline{v} \in M, \lambda \in[0,1]$ we have that

$$
e[\lambda \underline{u}+(1-\lambda) \underline{v}] \geq \lambda e(\underline{u})+(1-\lambda) e(\underline{v}) .
$$

Moreover, if $\lambda \in(0,1)$ we have equality if and only if $\underline{u}=\underline{v}$.

Proof. We begin with the inequality

$$
\|\underline{u}\|^{2}+\|\underline{v}\|^{2} \geq 2\|\underline{u}\|\|\underline{v}\| \text {. }
$$

Hence,

$$
1+\|\underline{u}\|^{2}\|\underline{v}\|^{2}-2\|\underline{u}\|\|\underline{v}\| \geq 1-\|\underline{u}\|^{2}-\|\underline{v}\|^{2}+\|\underline{u}\|^{2}\|\underline{v}\|^{2}=\left(1-\|\underline{u}\|^{2}\right)\left(1-\|\underline{v}\|^{2}\right)=e(\underline{u})^{2} e(\underline{v})^{2} .
$$

Taking the square root gives

$$
1-\|\underline{u}\|\|\underline{v}\| \geq e(\underline{u}) e(\underline{v})
$$

It follows that

$$
1-2 \lambda(1-\lambda)\|\underline{u}\|\|\underline{v}\| \geq 1-2 \lambda(1-\lambda)+2 \lambda(1-\lambda) e(\underline{u}) e(\underline{v})=\lambda^{2}+(1-\lambda)^{2}+2 \lambda(1-\lambda) e(\underline{u}) e(\underline{v}) .
$$

Hence,

$$
\begin{aligned}
1-[\lambda\|\underline{u}\|+(1-\lambda)\|\underline{v}\|]^{2} & =1-\lambda^{2}\|\underline{u}\|^{2}-(1-\lambda)^{2}\|\underline{v}\|^{2}-2 \lambda(1-\lambda)\|\underline{u}\|\|\underline{v}\| \\
& \geq \lambda^{2}\left(1-\|\underline{u}\|^{2}\right)+(1-\lambda)^{2}\left(1-\|\underline{v}\|^{2}\right)+2 \lambda(1-\lambda) e(\underline{u}) e(\underline{v})=[\lambda e(\underline{u})+(1-\lambda) e(\underline{v})]^{2} .
\end{aligned}
$$

Taking the square root and applying Schwarz's inequality gives

$$
\begin{aligned}
e[\underline{\lambda} \underline{u}+(1-\lambda) \underline{v}] & \left.=\left[1-\|\lambda \underline{u}+(1-\lambda) \underline{v}\|^{2}\right]^{1 / 2}=\left[1-\lambda^{2}\|\underline{u}\|^{2}-(1-\lambda)^{2}\|\underline{v}\|^{2}-2 \lambda(1-\lambda) \underline{u}, \underline{v}\right\rangle\right]^{1 / 2} \\
& \geq\left[1-\lambda^{2}\|\underline{u}\|^{2}-(1-\lambda)^{2}\|\underline{v}\|^{2}-2 \lambda(1-\lambda)\|\underline{u}\|\|\underline{v}\|\right]^{1 / 2}=\left[1-(\lambda\|\underline{u}\|+(1-\lambda)\|\underline{v}\|)^{2}\right]^{1 / 2} \\
& \geq \lambda e(\underline{u})+(1-\lambda) e(\underline{v}) .
\end{aligned}
$$


If we have equality, there is equality in Schwarz's inequality. This implies that $\underline{u}=a \underline{v}$ for some $a \in \mathbb{R}$. It follows that $\underline{u}=\underline{v}$.

Corollary 3. If $\underline{u}, \underline{v} \in M, \lambda \in(0,1)$ and $\underline{u} \neq \underline{v}$ then

$$
e[\lambda \underline{u}+(1-\lambda) \underline{v}]>\lambda e(\underline{u})+(1-\lambda) e(\underline{v}) .
$$

We define $M \times M$ to be the set of probability measures on $\mathbb{N} \times \mathbb{N}$. Thus $\underline{u} \in M \times M$ if $\underline{u}=\left\{u_{i j}: i, j \in \mathbb{N}\right\}, u_{i j} \geq 0$, $\sum u_{i j}=1$. As before, the entanglement number of $\underline{u}$ is defined by

$$
e(\underline{u})=\left(1-\|\underline{u}\|^{2}\right)^{1 / 2}=\left(1-\sum u_{i j}^{2}\right)^{1 / 2} .
$$

We also have that $e(\underline{u})=0$ if and only if $\underline{u}$ is a point measure with $u_{i j}=1$ for some $i, j \in \mathbb{N}$. If $\underline{v}, \underline{w} \in M$ we define $\underline{u}=\underline{v} \times \underline{w} \in M \times M$ by $u_{i j}=v_{i} w_{j}$. We say that $\underline{u} \in M \times M$ is factorized if $\underline{u}=\underline{v} \times \underline{w}$ for some $\underline{u}, \underline{v} \in M$. If $\underline{u}$ is not factorized, we say that $\underline{u}$ is entangled. It is easy to check that $\underline{u}$ is factorized if and only if for all $i, j \in \mathbb{N}$ we have [10]

$$
u_{i j}=\sum_{j} u_{i j} \sum_{i} u_{i j} .
$$

Note that if $e(\underline{u})=0$, then $\underline{u}$ is factorized. However, the converse does not hold because there are factorized $\underline{u}$ that are not point measures. For a quantum state $\psi$, we shall show that $e(\psi)=0$ if and only if $\psi$ is factorized and this will be an important difference between the quantum theory and this classical theory. It should be pointed out that $e(\psi)$ and factorization of $\psi$ are different in the quantum case, however, the analogy is similar.

Example 2. (a) Let $\underline{u} \in M \times M$ be defined by $u_{11}=1 / 2$, $u_{12}=1 / 2$. Then $e(\underline{u})=1 / \sqrt{2}$ and $\underline{u}=\underline{v} \times \underline{w}$ where $v_{1}=1, w_{1}=1 / 2, w_{2}=1 / 2$. Thus, $\underline{u}$ is factorized. (b) Let $\underline{u} \in M \times M$ be defined by $u_{11}=1 / 3, u_{12}=1 / 3$, $u_{22}=1 / 3$. Then $\sum u_{1 j}=2 / 3, \sum u_{i 1}=1 / 3$ and $\frac{1}{3} \neq \frac{2}{9}$ so (2) does not hold. Hence, $\underline{u}$ is entangled and we have $e(\underline{u})=\sqrt{2 / 3}$.

\section{Context Coefficients}

This section discusses the quantum statistics of operators. The basic framework for traditional quantum mechanics is a complex Hilbert space $H$. For simplicity, we shall assume that $\operatorname{dim} H<\infty$. Although this is a restriction, it is adequate for descriptions of quantum computation and information theory [1-3]. A pure state is represented by a one-dimensional projection $P$ on $H$. Since $P$ is onedimensional, we can describe $P$ by a unit vector $\phi$ in its range and write $P=P_{\phi}=|\phi\rangle\langle\phi|$. We also call $\phi$ a vector state (or state vector). A context for a quantum system is a set of mutually orthogonal projections $P_{\phi_{i}}$ on $H$ such that $\sum P_{\phi_{i}}=I$. Equivalently, a context can be described by the corresponding orthonormal basis $\left\{\phi_{i}\right\}$ of vector states. A context can be thought of as a complete set of minimal sharp events. We then see that there are an infinite uncountable number of contexts for a quantum system. This is in contrast to the classical systems described by $\mathbb{N}$ in Section 1. In that case, the minimal sharp events are just the points in $\mathbb{N}$ so the only context is $\mathbb{N}$ itself.

Let $\mathcal{L}(H)$ be the set of linear operators on $H$. The elements of $\mathcal{L}(H)$ are used to describe states, observables, symmetries and dynamics of the quantum system. If $A \in \mathcal{L}(H)$, we define the positive operator $|A|$ by $|A|=$ $\left(A^{*} A\right)^{1 / 2}$. A state is an operator $\rho \in \mathcal{L}(H)$ such that $\rho \geq 0$ and $\operatorname{tr}(\rho)=1$. Of course, a pure state is a specific type of state. We denote the set of states on $H$ by $\mathcal{S}(H)$. Any state has a spectral resolution $\rho=\sum \lambda_{i} P_{i}$ where $P_{i}$ are mutually orthogonal pure states, $\lambda_{i} \geq 0, \sum \lambda_{i}=1$. If $\rho \in \mathcal{S}(H)$ and $A \in \mathcal{L}(H)$, then the $\rho$-expectation of $A$ is $E_{\rho}(A)=\operatorname{tr}(\rho A)$ and the $\rho$-variance of $A$ is

$$
V_{\rho}(A)=E_{\rho}\left[\left|A-E_{\rho}(A) I\right|^{2}\right] .
$$

In particular, for a pure state $P_{\phi}$ we have that

$$
E_{\phi}(A)=E_{P_{\phi}}(A)=\langle\phi, A \phi\rangle
$$

and

$$
V_{\phi}(A)=V_{P_{\phi}}(A)=\left\langle\phi,|A-\langle\phi, A \phi\rangle I|^{2} \phi\right\rangle .
$$

The complex vector space $\mathcal{L}(H)$ becomes a Hilbert space under the Hilbert-Schmidt inner product $\langle A, B\rangle=$ $\operatorname{tr}\left(A^{*} B\right)[2,3]$. The Hilbert-Schmidt norm becomes

$$
\|A\|=\left[\operatorname{tr}\left(A^{*} A\right)\right]^{1 / 2}=\left[\operatorname{tr}\left(|A|^{2}\right)\right]^{1 / 2} .
$$

Theorem 4. (a) $V_{\rho}(A)=E_{\rho}\left(|A|^{2}\right)-\left|E_{\rho}(A)\right|^{2}$. (b) $\left|E_{\rho}(A)\right|^{2} \leq E_{\rho}\left(|A|^{2}\right)$ and $V_{\rho}(A)=0$ if and only if $A \rho^{1 / 2}=c \rho^{1 / 2}$ for some $c \in \mathbb{C}$.

Proof. The following computation proves (a).

$$
\begin{aligned}
V_{\rho}(A) & =\operatorname{tr}\left[\rho\left(A-E_{\rho}(A) I\right)^{*}\left(A-E_{\rho}(A) I\right)\right] \\
& =\operatorname{tr}\left[\rho\left(A^{*}-\overline{E_{\rho}(A) I}\right)\left(A-E_{\rho}(A) I\right)\right] \\
& =\operatorname{tr}\left[\rho\left(|A|^{2}-\overline{E_{\rho}(A)} A-E_{\rho}(A) A^{*}+\left|E_{\rho}(A)\right|^{2} I\right)\right] \\
& =E_{\rho}\left(|A|^{2}\right)-2\left|E_{\rho}(A)\right|^{2}+\left|E_{\rho}(A)\right|^{2} \\
& =E_{\rho}\left(|A|^{2}\right)-\left|E_{\rho}(A)\right|^{2}
\end{aligned}
$$

(b) Since $V_{\rho}(A) \geq 0$ we have that $\left|E_{\rho}(A)\right|^{2} \leq E_{\rho}\left(|(A)|^{2}\right)$. By (a) we have $V_{\rho}(A)=0$ if and only if 
$\left|E_{\rho}(A)\right|^{2}=E_{\rho}\left(|A|^{2}\right)$. In terms of the Hilbert-Schmidt inner product, we have that $V_{\rho}(A)=0$ if and only if

$$
\begin{aligned}
\left\|A \rho^{1 / 2}\right\|^{2} & =\operatorname{tr}\left[\left(A \rho^{1 / 2}\right)^{*} A \rho^{1 / 2}\right] \\
& =\operatorname{tr}\left(\rho^{1 / 2} A^{*} A \rho^{1 / 2}\right) \\
& =\operatorname{tr}\left(\rho A^{*} A\right) \\
& =E_{\rho}\left(|A|^{*}\right)=\left|E_{\rho}(A)\right|^{2} \\
& =|\operatorname{tr}(\rho A)|^{2} \\
& =\left|\operatorname{tr}\left(\rho^{1 / 2} A \rho^{1 / 2}\right)\right|^{2} \\
& =\left|\left\langle\rho^{1 / 2}, A \rho^{1 / 2}\right\rangle\right|^{2} .
\end{aligned}
$$

Since $\operatorname{tr}(\rho)=1$ we have that $\left\|\rho^{1 / 2}\right\|=1$. Hence,

$$
\left|\left\langle\rho^{1 / 2}, A \rho^{1 / 2}\right\rangle\right|=\left\|\rho^{1 / 2}\right\|\left\|A \rho^{1 / 2}\right\| .
$$

Since we have equality in Schwarz's inequality, we conclude that $A \rho^{1 / 2}=c \rho^{1 / 2}$ for some $c \in \mathbb{C}$.

Corollary 5. If $\phi$ is a vector state, then

$$
V_{\phi}(A)=\left\langle\phi,|A|^{2} \phi\right\rangle-|\langle\phi, A \phi\rangle|^{2}
$$

and $V_{\phi}(A)=0$ if and only if $A \phi=c \phi$ for some $c \in \mathbb{C}$; that is, $\phi$ is an eigenvector of $A$ with eigenvalue $c$.

A context given by an orthonormal basis $\mathcal{A}=\left\{\phi_{i}\right\}$ can be thought of as giving a partial view of a quantum system. In order to obtain a total view we must consider various contexts [11, 12]. We say that $A \in \mathcal{L}(H)$ is measurable with respect to $\mathcal{A}$ if $A P_{\phi_{i}}=P_{\phi_{i}} A$ for every $i$. In this case, $\phi_{i}$ is an eigenvector of $A$ with eigenvalue $\left\langle\phi_{i}, A \phi_{i}\right\rangle=E_{\phi_{i}}(A)$. The only operators accurately described by $\mathcal{A}$ are the operators that are measurable with respect to $\mathcal{A}[11,12]$. We define the context coefficient of $A$ with respect to $\mathcal{A}$ by

$$
c_{\mathcal{A}}(A)=\left[\sum V_{\phi_{i}}(A)\right]^{1 / 2} .
$$

It follows from Corollary 5 that $c_{\mathcal{A}}(A)=0$ if and only if $A$ is measurable with respect to $\mathcal{A}$. We can consider $c_{\mathcal{A}}(A)$ as an indicator of how close $A$ is to being measurable with respect to $\mathcal{A}$. We also see that $A$ is normal $\left(A A^{*}=A^{*} A\right)$ if and only if $c_{\mathcal{A}}(A)=0$ for some context $\mathcal{A}$. For any $A \in \mathcal{L}(H)$ and context $\mathcal{A}=\left\{\phi_{i}\right\}$ we can write

$$
A=\sum_{i}\left\langle\phi_{i}, A \phi_{i}\right\rangle\left|\phi_{i}\right\rangle\left\langle\phi_{i}\left|+\sum_{i \neq j}\left\langle\phi_{i}, A \phi_{j}\right\rangle\right| \phi_{i}\right\rangle\left\langle\phi_{j}\right| .
$$

We define the linear maps $L_{\mathcal{A}}, R_{\mathcal{A}}: \mathcal{L}(H) \rightarrow \mathcal{L}(H)$ by

$$
\begin{aligned}
& L_{\mathcal{A}}(A)=\sum_{i}\left\langle\phi_{i}, A \phi_{i}\right\rangle\left|\phi_{i}\right\rangle\left\langle\phi_{i}\right| \\
& R_{\mathcal{A}}(A)=\sum_{i \neq j}\left\langle\phi_{i}, A \phi_{j}\right\rangle\left|\phi_{i}\right\rangle\left\langle\phi_{j}\right|
\end{aligned}
$$

and call $L_{\mathcal{A}}$ the context map and $R_{\mathcal{A}}$ the residual map. Thus, $A=L_{\mathcal{A}}(A)+R_{\mathcal{A}}(A)$. Notice that $L_{\mathcal{A}}$ maps selfadjoint operators to self-adjoint operators, positive operators to positive operators and states to states. In fact, $L_{\mathcal{A}}$ is a completely positive map [2,3,12] and is an example of a quantum channel [2,3]. Also $L_{\mathcal{A}}(A)$ is measurable with respect to $\mathcal{A}$ and $A$ is measurable with respect to $\mathcal{A}$ if and only if $L_{\mathcal{A}}(A)=A$ or equivalently $R_{\mathcal{A}}(A)=0$. We remind the reader that $\|B\|$ stands for the Hilbert-Schmidt norm of $B \in \mathcal{L}(H)$.

Theorem 6. For every $A \in \mathcal{L}(H)$ and context $\mathcal{A}=\left\{\phi_{i}\right\}$ we have that $\left\|R_{\mathcal{A}}(A)\right\|=c_{\mathcal{A}}(A)$.

Proof. Since

$$
\begin{aligned}
R_{\mathcal{A}}(A)^{*} R_{\mathcal{A}}(A) & =\sum_{i \neq j}\left\langle A \phi_{j}, \phi_{i}\right\rangle\left|\phi_{j}\right\rangle\left\langle\phi_{i}\left|\cdot \sum_{r \neq s}\left\langle\phi_{r}, A \phi_{s}\right\rangle\right| \phi_{r}\right\rangle\left\langle\phi_{s}\right| \\
& =\sum_{\substack{i, j, s \\
i \neq s, j}}\left\langle A \phi_{j}, \phi_{i}\right\rangle\left\langle\phi_{i}, A \phi_{s}\right\rangle\left|\phi_{j}\right\rangle\left\langle\phi_{s}\right|
\end{aligned}
$$

we conclude that

$$
\begin{aligned}
\left\|R_{\mathcal{A}}(A)\right\|^{2} & =\operatorname{tr}\left[R_{\mathcal{A}}(A)^{*} R_{\mathcal{A}}(A)\right] \\
& =\sum_{i \neq k}\left\langle A \phi_{k}, \phi_{i}\right\rangle\left\langle\phi_{i}, A \phi_{k}\right\rangle \\
& =\sum_{i, k}\left\langle A \phi_{k}, \phi_{i}\right\rangle\left\langle\phi_{i}, A \phi_{k}\right\rangle-\sum_{i}\left\langle A \phi_{i}, \phi_{i}\right\rangle\left\langle\phi_{i}, A \phi_{i}\right\rangle \\
& =\sum_{k}\left\langle A \phi_{k}, A \phi_{k}\right\rangle-\sum_{i}\left|\left\langle\phi_{i}, A \phi_{i}\right\rangle\right|^{2} \\
& =\sum_{k}\left(\left\langle\phi_{k},|A|^{2} \phi_{k}\right\rangle-\left|\left\langle\phi_{k}, A \phi_{k}\right\rangle\right|^{2}\right) \\
& =\sum_{k} V_{\phi_{k}}(A)=c_{\mathcal{A}}(A)^{2} .
\end{aligned}
$$

It follows from Theorem 6 that $c_{\mathcal{A}}(A)=\left\|A-L_{\mathcal{A}}(A)\right\|$ so that $c_{\mathcal{A}}(A)$ is a measure of the closeness of $A$ to $L_{\mathcal{A}}(A)$. Of course, $c_{\mathcal{A}}(A)=0$ if and only if $A=L_{\mathcal{A}}(A), c_{\mathcal{A}}(\alpha A)=$ $|\alpha| c_{\mathcal{A}}(A)$ and

$$
c_{\mathcal{A}}(A+B) \leq c_{\mathcal{A}}(A)+c_{\mathcal{A}}(B) .
$$

Observe that $L_{\mathcal{A}}(A)$ is always normal with eigenvalues $\left\langle\phi_{i}, A \phi_{i}\right\rangle$ and corresponding eigenvectors $\phi_{i}$. In general $R_{\mathcal{A}}(A)$ need not be normal and even when it is, its eigenstructure can be difficult to analyze except in two simple but important cases. One is when $\operatorname{dim} H=2$ and the other when $\left\langle\phi_{i}, A \phi_{j}\right\rangle=\alpha$ for all $i \neq j$.

Example 3. Suppose $\operatorname{dim} H=2$ and $R_{\mathcal{A}}(A)$ is normal. We can write

$$
\begin{aligned}
R_{\mathcal{A}}(A) & =\left\langle\phi_{1}, A \phi_{2}\right\rangle\left|\phi_{1}\right\rangle\left\langle\phi_{2}\left|+\left\langle\phi_{2}, A \phi_{1}\right\rangle\right| \phi_{2}\right\rangle\left\langle\phi_{1}\right| \\
& =a\left|\phi_{1}\right\rangle\left\langle\phi_{2}|+b| \phi_{2}\right\rangle\left\langle\phi_{1}\right| .
\end{aligned}
$$


We assume that $a, b \neq 0$ because otherwise the situation is trivial. It is easy to check that $R_{\mathcal{A}}(A)$ is normal if and only if $|a|=|b|$ in which case $a=|a| e^{i \theta}, b=|a| e^{i \phi}, \theta, \phi \in \mathbb{R}$. Then the eigenvalues of $R_{\mathcal{A}}(A)$ are

$$
\lambda_{1}=|a| e^{i(\theta+\phi) / 2}, \quad \lambda_{2}=-|a| e^{i(\theta+\phi) / 2}
$$

with corresponding eigenvectors

$$
\begin{aligned}
& \psi_{1}=\frac{1}{\sqrt{2}}\left[\phi_{1}+e^{i(\phi-\theta)} \phi_{2}\right], \\
& \psi_{2}=\frac{1}{\sqrt{2}}\left[-e^{i(\theta-\phi) / 2} \phi_{1}+\phi_{2}\right] .
\end{aligned}
$$

Example 4. Suppose $\operatorname{dim} H=n$ and $\left\langle\phi_{i}, A \phi_{j}\right\rangle=\alpha \neq 0$, $i, j=1, \ldots, n$. We then have that

$$
R_{\mathcal{A}}(A)=\alpha \sum_{i \neq j}\left|\phi_{i}\right\rangle\left\langle\phi_{j}\right| .
$$

It follows that $R_{\mathcal{A}}(A)^{*}=\frac{\bar{\alpha}}{\alpha} R_{\mathcal{A}}(A)$ so $R_{\alpha}(A)$ is normal. For the rest of this example, we assume that $\alpha=1$ and $\alpha$ can be multiplied later if needed. First note that $R_{\mathcal{A}}(A) \phi_{k}=$ $\sum_{i \neq k} \phi_{i}$. Letting $\psi=\frac{1}{\sqrt{n}} \sum_{k=1}^{n} \phi_{k}$, it follows that

$$
\begin{aligned}
R_{\mathcal{A}}(A) \psi & =\frac{1}{\sqrt{n}} \sum_{i \neq j}\left|\phi_{i}\right\rangle\left\langle\phi_{k}\right| \sum_{k=1}^{n} \phi_{k} \\
& =\frac{1}{\sqrt{n}} \sum_{k=1}^{n} \sum_{i \neq k} \phi_{i} \\
& =\frac{n-1}{\sqrt{n}} \sum_{k=1}^{n} \psi_{k}=(n-1) \psi .
\end{aligned}
$$

Hence, $\psi$ is a normalized eigenvector of $R_{\mathcal{A}}(A)$ with eigenvalue $n-1$. We will show that the other $n-1$ eigenvectors of $R_{\mathcal{A}}(A)$ all have eigenvalue -1 so -1 has multiplicity $n-1$. The simplest way to show this is to examine the first few cases and to observe the resulting pattern. When $n=2$, we have that $\frac{1}{\sqrt{2}}\left(\phi_{1}-\phi_{2}\right)$ is an eigenvector with eigenvalue -1 . When $n=4, \frac{1}{\sqrt{2}}\left(\phi_{1}-\phi_{2}\right)$, $\frac{1}{\sqrt{2}}\left(\phi_{3}-\phi_{4}\right), \frac{1}{2}\left(\phi_{1}+\phi_{2}-\phi_{3}-\phi_{4}\right)$ are eigenvectors with eigenvalue -1 . When $n=6, \frac{1}{\sqrt{2}}\left(\phi_{1}-\phi_{2}\right), \frac{1}{\sqrt{2}}\left(\phi_{3}-\phi_{4}\right)$, $\frac{1}{\sqrt{2}}\left(\phi_{5}-\phi_{6}\right), \frac{1}{2}\left(\phi_{3}+\phi_{4}-\phi_{5}-\phi_{6}\right), \frac{1}{\sqrt{8}}\left(2 \phi_{1}+2 \phi_{2}-\phi_{3}-\right.$ $\left.\phi_{4}-\phi_{5}-\phi_{6}\right)$ are eigenvectors with eigenvalue -1 . When $n=3, \frac{1}{\sqrt{2}}\left(\phi_{1}-\phi_{2}\right), \frac{1}{2}\left(\phi_{1}+\phi_{2}-2 \phi_{3}\right)$ are eigenvectors with eigenvalue -1 . When $n=5, \frac{1}{\sqrt{2}}\left(\phi_{1}-\phi_{2}\right), \frac{1}{\sqrt{2}}\left(\phi_{3}-\phi_{4}\right)$, $\frac{1}{2}\left(\phi_{1}+\phi_{2}-\phi_{3}-\phi_{4}\right), \frac{1}{\sqrt{8}}\left(\phi_{1}+\phi_{2}+\phi_{3}+\phi_{4}-4 \phi_{5}\right)$ are eigenvectors with eigenvalue -1 . In summary, we have the following result.

Theorem 7. Let $R_{\mathcal{A}}(A)=\sum_{i \neq j}\left|\phi_{i}\right\rangle\left\langle\phi_{j}\right|$ and let $\operatorname{dim} H=n$. Then $R_{\mathcal{A}}(A)$ has eigenvalue $n-1$ with corresponding eigenvector $\psi=\frac{1}{\sqrt{n}} \sum_{k=1}^{n} \phi_{k}$ and $R_{\mathcal{A}}(A)$ has eigenvalue -1 with multiplicity $n-1$ and the corresponding eigenvectors form an orthonormal basis for $\{\psi\}^{\perp}$.

\section{Entanglement}

We now incorporate the two previous sections to develop a general theory of quantum entanglement. We restrict attention to bipartite systems and leave multipartite systems for later work. Let $H_{1}, H_{2}$ be finite dimensional complex Hilbert spaces and let $H=H_{1} \otimes H_{2}$. A state $\rho \in \mathcal{S}(H)$ is factorized if there exist states $\rho_{1} \in \mathcal{S}\left(H_{1}\right)$, $\rho_{2} \in \mathcal{S}\left(H_{2}\right)$ such that $\rho=\rho_{1} \otimes \rho_{2}$. A state $\rho \in \mathcal{S}(H)$ is separable if $\rho$ can be written as a convex combination $\rho=\sum \lambda_{i} \rho_{i} \otimes \sigma_{i}$ of factorized states. If $\rho$ is not separable, it is entangled. Also, we say that a vector state $\psi \in H$ is factorized if there exist vector states $\phi_{1} \in H_{1}, \phi_{2} \in H_{2}$ such that $\psi=\phi_{1} \otimes \phi_{2}$. If $\psi$ is not factorized, then $\psi$ is entangled. The following lemma summarizes some known properties of factorized states [2]. We include the proofs for completeness.

Lemma 8. (a) A pure state $|\eta\rangle\langle\eta| \in \mathcal{S}(H)$ is factorized if and only if the vector state $\eta$ is factorized (b) A pure state $|\eta\rangle\langle\eta| \in \mathcal{S}(H)$ is separable if and only if $|\eta\rangle\langle\eta|$ is factorized.

Proof. (a) If $\eta \in H$ is factorized, then $\eta=\eta_{1} \otimes \eta_{2}$, $\eta_{i} \in H_{i}, i=1,2$. Hence,

$$
|\eta\rangle\left\langle\eta|=| \eta_{1} \otimes \eta_{2}\right\rangle\left\langle\eta_{1} \otimes \eta_{2}|=| \eta_{1}\right\rangle\left\langle\eta_{1}|\otimes| \eta_{2}\right\rangle\left\langle\eta_{2}\right|
$$

so $|\eta\rangle\langle\eta|$ is factorized. Conversely, if $|\eta\rangle\langle\eta|$ is factorized, then $|\eta\rangle\langle\eta|=\rho_{1} \otimes \rho_{2}, \rho_{i} \in \mathcal{S}(H), i=1,2$. Since

$$
\rho_{1}^{2} \otimes \rho_{2}^{2}=\left(\rho_{1} \otimes \rho_{2}\right)^{2}=\rho_{1} \otimes \rho_{2}
$$

we have that $\rho_{1}^{2}=\rho_{1}$ and $\rho_{2}^{2}=\rho_{2}$ so $\rho_{1}$ and $\rho_{2}$ are projections. Since

$$
\operatorname{tr}\left(\rho_{1}\right) \operatorname{tr}\left(\rho_{2}\right)=\operatorname{tr}\left(\rho_{1} \otimes \rho_{2}\right)=1
$$

we have that $\operatorname{tr}\left(\rho_{1}\right)=\operatorname{tr}\left(\rho_{2}\right)=1$ so $\rho_{1}$ and $\rho_{2}$ are pure states. Hence, $\rho_{1}=\left|\phi_{1}\right\rangle\left\langle\phi_{1}\left|, \rho_{2}=\right| \phi_{2}\right\rangle\left\langle\phi_{2}\right|, \phi_{i} \in H_{i}$, $i=1,2$ and we have that

$$
|\eta\rangle\left\langle\eta|=| \phi_{1}\right\rangle\left\langle\phi_{1}|\otimes| \phi_{2}\right\rangle\left\langle\phi_{2}|=| \phi_{1} \otimes \phi_{2}\right\rangle\left\langle\phi_{1} \otimes \phi_{2}\right| .
$$

Thus, $\eta=\phi_{1} \otimes \phi_{2}$ so $\eta$ is factorized.

(b) If $|\eta\rangle\langle\eta|$ is factorized, then clearly $|\eta\rangle\langle\eta|$ is separable. Conversely, if $|\eta\rangle\langle\eta|$ is separable, then there are $\lambda_{i}>0$ with

$$
|\eta\rangle\langle\eta|=\sum_{i=1}^{n} \lambda_{i} \rho_{i} \otimes \sigma_{i} .
$$

We concluded that $\lambda_{i} \rho_{i} \otimes \sigma_{i} \leq|\eta\rangle\langle\eta|$ and since $|\eta\rangle\langle\eta|$ is one-dimensional we have that $\lambda_{i} \rho_{i} \otimes \sigma_{i}=\lambda|\eta\rangle\langle\eta|$ for some $\lambda \in[0,1]$. Taking the trace gives $\lambda_{i}=\lambda$ so $|\eta\rangle\langle\eta|=\rho_{i} \otimes \sigma_{i}$, $i=1, \ldots, n$. Therefore, $|\eta\rangle\langle\eta|=\rho_{1} \otimes \sigma_{1}$ so $|\eta\rangle\langle\eta|$ is factorized. 
Let $\mathcal{A}=\left\{\phi_{i}\right\}, \mathcal{B}=\left\{\psi_{i}\right\}$ be orthonormal bases (contexts) for $H_{1}$ and $H_{2}$, respectively. If $\underline{\lambda} \in M$ is a probability measure, we call $(\underline{\lambda}, \mathcal{A}, \mathcal{B})$ an entanglement and we call $(M, \mathcal{A}, \mathcal{B})$ an entanglement system. We assume without loss of generality that $\operatorname{dim} H_{1}=\operatorname{dim} H_{2}=n$. We can do this because if $\operatorname{dim} H_{1}<\operatorname{dim} H_{2}$, say, then we can enlarge $H_{1}$ to $\operatorname{dim} H_{2}$ and no harm is done. Moreover, we assume that $\operatorname{supp}(\underline{\lambda}) \subseteq\{1,2, \ldots, n\}$. Corresponding to an entanglement $E=(\underline{\lambda}, \mathcal{A}, \mathcal{B})$ we have a vector state

$$
\psi_{E}=\sum \sqrt{\lambda_{i}} \phi_{i} \otimes \psi_{i} \in H_{1} \otimes H_{2}
$$

a pure state $P_{E}=P_{\psi_{E}}$, a separable state

$$
\rho_{E}=\sum \lambda_{i} P_{\phi_{i} \otimes \psi_{i}}=\sum \lambda_{i} P_{\phi_{i}} \otimes P_{\psi_{i}}
$$

and an entanglement operator

$$
\begin{aligned}
B_{E} & =\sum_{i \neq j} \sqrt{\lambda_{i} \lambda_{j}}\left|\phi_{i} \otimes \psi_{i}\right\rangle\left\langle\phi_{j} \otimes \psi_{j}\right| \\
& =\sum_{i \neq j} \sqrt{\lambda_{i} \lambda_{j}}\left|\phi_{i}\right\rangle\left\langle\phi_{j}|\otimes| \psi_{i}\right\rangle\left\langle\psi_{j}\right| .
\end{aligned}
$$

From Section 1 , since $\underline{\lambda} \in M$ we have the entanglement number $e(\underline{\lambda})$. We use this to define the entanglement number

$$
e\left(\psi_{E}\right)=e\left(P_{E}\right)=e(\underline{\lambda}) .
$$

Conversely, if $\psi \in H_{1} \otimes H_{2}$ is a vector state, then there exists a Schmidt decomposition consisting of an entanglement $(\lambda, \mathcal{A}, \mathcal{B})$ where $\lambda \in M$ is unique and $\psi=$ $\sum \sqrt{\lambda_{i}} \phi_{i} \otimes \psi_{i}[2,3,6]$. In this way, any vector state $\psi$ determines an entanglement $E=(\underline{\lambda}, \mathcal{A}, \mathcal{B})$ so that $\psi=\psi_{E}$ although $\mathcal{A}$ and $\mathcal{B}$ need not be unique. It is easy to check that

$$
P_{E}=\left|\psi_{E}\right\rangle\left\langle\psi_{E}\right|=\rho_{E}+B_{E}
$$

and $B_{E}$ is a self-adjoint, traceless operator. We consider $\rho_{E}$ as the non-entangled part of $P_{E}$ and $B_{E}$ as describing the entangled part. Letting $\mathcal{D}=\mathcal{A} \otimes \mathcal{B}=\left\{\phi_{i} \otimes \psi_{j}\right\}$ be the corresponding orthonormal basis (context) for $H=$ $H_{1} \otimes H_{2}$ we have that $\rho_{E}=L_{\mathcal{D}}\left(P_{E}\right)$ and $B_{E}=R_{\mathcal{D}}\left(P_{E}\right)$ where $L_{\mathcal{D}}$ and $R_{\mathcal{D}}$ are the context map and residual map of Section 2 .

Considering the Hilbert-Schmidt norm $\left\|B_{E}\right\|$ we see that $\left\|B_{E}\right\|=\left\|P_{E}-\rho_{E}\right\|$ gives a measure of the entanglement of $P_{E}$. Thus, if $\left\|B_{E}\right\|$ is small, then $P_{E}$ is close to $\rho_{E}$ and is less entangled and when $\left\|B_{E}\right\|$ is large, then $P_{E}$ is more entangled. The next result shows that our three entanglement measures coincide.

Theorem 9. $c_{\mathcal{D}}\left(B_{E}\right)=\left\|B_{E}\right\|=e\left(\psi_{E}\right)$

Proof. It follows from Theorem 6 that $c_{\mathcal{D}}\left(B_{E}\right)=\left\|B_{E}\right\|$. To show that $\left\|B_{E}\right\|=e\left(\psi_{E}\right)$ we have that

$$
\begin{aligned}
B_{E}^{2} & =\left[\sum_{i \neq j} \sqrt{\lambda_{i} \lambda_{j}}\left|\phi_{i} \otimes \psi_{i}\right\rangle\left\langle\phi_{j} \otimes \psi_{j}\right|\right]\left[\sum_{r \neq s} \sqrt{\lambda_{r} \lambda_{s}}\left|\phi_{r} \otimes \psi_{r}\right\rangle\left\langle\phi_{s} \otimes \psi_{s}\right|\right] \\
& =\sum_{i \neq j} \sum_{s} \sqrt{\lambda_{i} \lambda_{s}}\left|\phi_{i} \otimes \psi_{i}\right\rangle\left\langle\phi_{s} \otimes \psi_{s}\left|=\sum_{i, s}\left(1-\lambda_{i}\right) \sqrt{\lambda_{i} \lambda_{s}}\right| \phi_{i} \otimes \psi_{i}\right\rangle\left\langle\phi_{s} \otimes \psi_{s}\right| .
\end{aligned}
$$

Hence,

$$
\operatorname{tr}\left(B_{E}^{2}\right)=\sum\left(1-\lambda_{i}\right) \lambda_{i}=1-\sum \lambda_{i}^{2}=1-\|\underline{\lambda}\|^{2} .
$$

We conclude that

$$
\left\|B_{E}\right\|=\left[\operatorname{tr}\left(B_{E}^{2}\right)\right]^{1 / 2}=\sqrt{1-\|\underline{\lambda}\|^{2}}=e(\underline{\lambda})=e\left(\psi_{E}\right) .
$$

Let $E=(\underline{\alpha}, \mathcal{A}, \mathcal{B})$ and $F=(\beta, \mathcal{A}, \mathcal{B})$ be entanglements belonging to the same entanglement system $(M, \mathcal{A}, \mathcal{B})$. We have the corresponding vector states $\psi_{E}=\sum \sqrt{\alpha_{i}} \phi_{i} \otimes$ $\psi_{i}, \psi_{F}=\sum \sqrt{\beta_{i}} \phi_{i} \otimes \psi_{i}$. For $\lambda \in(0,1)$ we have the entanglement

$$
G=(\lambda \underline{\alpha}+(1-\lambda) \underline{\beta}, \mathcal{A}, \mathcal{B})
$$

and vector state

$$
\psi_{G}=\sum \sqrt{\lambda \alpha_{i}+(1-\lambda) \beta_{i}} \phi_{i} \otimes \psi_{i}
$$

By Theorem 2 we have that

$$
\begin{aligned}
e\left(\psi_{G}\right) & =e[\lambda \underline{\alpha}+(1-\lambda) \underline{\beta}] \\
& \geq \lambda e(\underline{\alpha})+(1-\lambda) e(\underline{\beta}) \\
& =\lambda e\left(\psi_{E}\right)+(1-\lambda) e\left(\psi_{F}\right) .
\end{aligned}
$$

Our entanglement number is related to entanglement robustness [6, 8, 9, 13-16], but there are important differences and the motivation is not the same. We leave a detailed comparison to later work.

Example 5. Let $H=\mathbb{C}^{2} \otimes \mathbb{C}^{2}$ and let $\psi \in H$ be a vector state. By the Schmidt decomposition, there are numbers $\lambda_{1}, \lambda_{2} \geq 0$ with $\lambda_{1}+\lambda_{2}=1$ and bases $\mathcal{A}=\left\{\phi_{1}, \phi_{2}\right\}$, $\mathcal{B}=\left\{\psi_{1}, \psi_{2}\right\}$ of $\mathbb{C}^{2}$ such that

$$
\psi=\sqrt{\lambda_{1}} \phi_{1} \otimes \psi_{1}+\sqrt{\lambda_{2}} \phi_{2} \otimes \psi_{2} .
$$


We have that $P_{\psi}=\rho_{\psi}+B_{\psi}$ where

$$
\begin{aligned}
& \rho_{\psi}=\lambda_{1}\left|\phi_{1} \otimes \psi_{1}\right\rangle\left\langle\phi_{1} \otimes \psi_{1}\left|+\lambda_{2}\right| \phi_{2} \otimes \psi_{2}\right\rangle\left\langle\phi_{2} \otimes \psi_{2}\right|, \\
& B_{\psi}=\sqrt{\lambda_{1} \lambda_{2}}\left[\left|\phi_{1} \otimes \psi_{1}\right\rangle\left\langle\phi_{2} \otimes \psi_{2}|+| \phi_{2} \otimes \psi_{2}\right\rangle\left\langle\phi_{1} \otimes \psi_{1}\right|\right] .
\end{aligned}
$$

We see that $\rho_{\psi}$ is a separable state and the entanglement operator $B_{\psi}$ is self-adjoint and traceless. The eigenvalues of $B_{\psi}$ are $0,0, \sqrt{\lambda_{1} \lambda_{2}},-\sqrt{\lambda_{1} \lambda_{2}}$. The corresponding eigenvectors are $\phi_{1} \otimes \psi_{2}, \phi_{2} \otimes \psi_{1}$ which are factorized and

$$
\frac{1}{\sqrt{2}}\left(\phi_{1} \otimes \psi_{1}+\phi_{2} \otimes \psi_{2}\right), \quad \frac{1}{\sqrt{2}}\left(\phi_{1} \otimes \psi_{1}-\phi_{2} \otimes \psi_{2}\right)
$$

which are entangled. The Hilbert-Schmidt norm of $B_{\psi}$ is

$$
\left\|B_{\psi}\right\|=\sqrt{2 \lambda_{1} \lambda_{2}}=e(\psi) .
$$

Example 6. Let $E=(M, \mathcal{A}, \mathcal{B})$ be an entanglement system with $\mathcal{A}=\left\{\phi_{i}\right\}, \mathcal{B}=\left\{\psi_{j}\right\}$. Consider the following vector states in $E$

$$
\begin{aligned}
\alpha & =\frac{1}{\sqrt{2}} \phi_{1} \otimes \psi_{1}+\frac{1}{\sqrt{2}} \phi_{2} \otimes \psi_{2} \\
\beta & =\frac{1}{\sqrt{3}} \phi_{1} \otimes \psi_{1}+\frac{1}{\sqrt{3}} \phi_{2} \otimes \psi_{2}+\frac{1}{\sqrt{3}} \phi_{3} \otimes \psi_{3} \\
\gamma & =\frac{1}{\sqrt{2}} \phi_{1} \otimes \psi_{1}+\frac{1}{\sqrt{3}} \phi_{2} \otimes \psi_{2}+\frac{1}{\sqrt{6}} \phi_{3} \otimes \psi_{3} \\
\delta & =\frac{1}{3} \phi_{1} \otimes \psi_{1}+\frac{1}{3} \phi_{2} \otimes \psi_{2}+\sqrt{\frac{7}{9}} \phi_{3} \times \psi_{3} .
\end{aligned}
$$

All of these states are entangled and as in Example 1 we have $e(\alpha)=1 / \sqrt{2}, e(\beta)=\sqrt{2 / 3}, e(\gamma)=\sqrt{11 / 18}$, $e(\delta)=\sqrt{30} / 9$ and we have that

$$
e(\delta)<e(\alpha)<e(\gamma)<e(\beta)
$$

Example 7. If $\mathcal{A}=\left\{\phi_{i}\right\}$ is an orthonormal basis for $H$, the corresponding symmetricantisymmetric basis for $H \otimes H$ is

$$
\mathcal{A}^{S A}=\left\{\phi_{i} \otimes \phi_{i}, \frac{1}{\sqrt{2}}\left(\phi_{i} \otimes \phi_{j}+\phi_{j} \otimes \phi_{i}\right), \frac{1}{\sqrt{2}}\left(\phi_{i} \otimes \phi_{j}-\phi_{j} \otimes \phi_{i}\right), i<j\right\} .
$$

The first two types are symmetric and the last type are antisymmetric. There are $n(n+1) / 2$ symmetric and $n(n-1) / 2$ antisymmetric states. The entanglement number for the first type is 0 and the others are $1 / \sqrt{2}$. We have that $P_{\phi_{i} \otimes \phi_{i}}=P_{\phi_{i}} \otimes P_{\phi_{i}}$ is factorized and

$$
\begin{aligned}
P_{\frac{1}{\sqrt{2}}\left(\phi_{i} \otimes \phi_{j}+\phi_{j} \otimes \phi_{i}\right)} & =\frac{1}{2}\left|\phi_{i}\right\rangle\left\langle\phi_{i}|\otimes| \phi_{j}\right\rangle\left\langle\phi_{j}\left|+\frac{1}{2}\right| \phi_{j}\right\rangle\left\langle\phi_{j}|\otimes| \phi_{i}\right\rangle\left\langle\phi_{i}\left|+\frac{1}{2}\right| \phi_{j}\right\rangle\left\langle\phi_{i}|\otimes| \phi_{i}\right\rangle\left\langle\phi_{j}\left|+\frac{1}{2}\right| \phi_{i}\right\rangle\left\langle\phi_{j}|\otimes| \phi_{j}\right\rangle\left\langle\phi_{i}\right| \\
& =\frac{1}{2} P_{\phi_{i}} \otimes P_{\phi_{j}}+\frac{1}{2} P_{\phi_{j}} \otimes P_{\phi_{i}}+\operatorname{Re}\left(\left|\phi_{j}\right\rangle\left\langle\phi_{i}|\otimes| \phi_{i}\right\rangle\left\langle\phi_{j}\right|\right) .
\end{aligned}
$$

We can write this as $A+B$ where $A$ is the separable state

$$
A=\frac{1}{2} P_{\phi_{i}} \otimes P_{\phi_{j}}+\frac{1}{2} P_{\phi_{j}} \otimes P_{\phi_{i}}
$$

and $B$ is the entanglement operator. We also have

$$
P_{\frac{1}{\sqrt{2}}\left(\phi_{i} \otimes \phi_{j}-\phi_{j} \otimes \phi_{i}\right)}=A-B .
$$

Example 8. Let $H=H_{1} \otimes H_{2}$ with $\operatorname{dim} H_{1}=\operatorname{dim} H_{2}=n$ and let $\psi \in H$ be the maximally entangled vector given by

$$
\psi=\frac{1}{\sqrt{n}} \sum \phi_{i} \otimes \psi_{i}
$$

where $\mathcal{A}=\left\{\phi_{i}\right\}, \mathcal{B}=\left\{\psi_{i}\right\}$ are orthonormal bases for $H_{1}, H_{2}$, respectively. Letting $\lambda_{i}=1 / n, i=1,2, \ldots, n$ and $E=(\underline{\lambda}, \mathcal{A}, \mathcal{B})$ we have that $\psi=\psi_{E}$ with corresponding pure state $P_{E}$ and entanglement operator

$$
B_{E}=\frac{1}{n} \sum\left|\phi_{i}\right\rangle\left\langle\phi_{j}|\otimes| \psi_{i}\right\rangle\left\langle\psi_{j}\right| \text {. }
$$

Letting $\mathcal{D}=\left\{\phi_{i} \otimes \psi_{j}\right\}$ be the resulting orthonormal basis for $H$ and $R_{\mathcal{D}}$ be the corresponding residual map we have $B_{E}=R_{\mathcal{D}}\left(P_{E}\right)$. It follows from Theorem 7 that the nonzero eigenvalues of $B_{E}$ are $1-\frac{1}{n}$ and $-\frac{1}{n}$. The eigenvalue $1-\frac{1}{n}$ has multiplicity 1 and corresponding eigenvector $\psi$ while the eigenvalue $-\frac{1}{n}$ has multiplicity $n-1$ whose eigenspace is the subspace of $H$ generated by $\left\{\phi_{i} \otimes \psi_{j}: i \neq j\right\}$ and orthogonal to $\phi$.

Until now we have considered the entanglement number for a pure state $P_{\phi}$. For the remainder of this article we shall discuss mixed states. If $\rho$ is a mixed state on $H$ that is not pure, then $\rho$ possesses an uncountably infinite number of decompositions $\rho=\sum \lambda_{i} P_{i}, \lambda_{i}>0, \sum \lambda_{i}=1$ where $P_{i}$ are pure states [2]. Also, $\rho$ has a spectral decomposition $\rho=\sum \mu_{i} Q_{i}, \mu_{i}>0, \sum \mu_{i}=1$, where $Q_{i}$ are mutually orthogonal pure states. The $\mu_{i}$ are the nonzero eigenvalues of $\rho$ and the ranges of $Q_{i}$ are the corresponding eigenvectors of $\rho$. The next example is based on Example 6.13 in [2].

Example 9. Let $H=\mathbb{C}^{2} \otimes \mathbb{C}^{2}$, let $\left\{\phi_{1}, \phi_{2}\right\}$ be an orthonormal basis for $\mathbb{C}^{2}$ and define $\phi=\frac{1}{\sqrt{2}}\left(\phi_{1}+\phi_{2}\right)$. We now 
consider the separable state

$$
\rho=\frac{1}{2}\left(|\phi \otimes \phi\rangle\left\langle\phi \otimes \phi|+| \phi_{1} \otimes \phi_{1}\right\rangle\left\langle\phi_{1} \otimes \phi_{1}\right|\right) .
$$

The eigenvalues of $\rho$ are 0 (multiplicity 2), 1/4 and 3/4. The eigenvectors for 0 are

$$
\begin{aligned}
& \psi_{1}=\frac{1}{\sqrt{2}}\left(\phi_{2}-\phi_{1}\right) \otimes \phi_{2}, \\
& \psi_{2}=\frac{1}{\sqrt{6}}\left[\left(\phi_{1}+\phi_{2}\right) \otimes \phi_{2}-2 \phi_{2} \otimes \phi_{1}\right] .
\end{aligned}
$$

The eigenvectors for $1 / 4$ and $3 / 4$ are

$$
\begin{aligned}
& \psi_{3}=\frac{1}{2 \sqrt{3}}\left[\left(3 \phi_{1}+\phi_{2}\right) \otimes \phi_{1}+\left(\phi_{1}+\phi_{2}\right) \otimes \phi_{2}\right] \\
& \psi_{4}=\frac{1}{2}\left[\left(\phi_{2}-\phi_{1}\right) \otimes \phi_{1}+\left(\phi_{1}+\phi_{2}\right) \otimes \phi_{2}\right] .
\end{aligned}
$$

The unique spectral decomposition of $\rho$ becomes

$$
\rho=\frac{1}{4} P_{\psi_{3}}+\frac{3}{4} P_{\psi_{4}} .
$$

Notice that $\psi_{3}$ and $\psi_{4}$ are entangled. This gives an example of a separable state whose unique spectral decomposition consists of entangled pure states.

Example 9 shows that a spectral decomposition cannot be used to determine an entanglement number for a mixed state. Indeed, in (4) since $\rho$ is separable the entanglement number for $\rho$ should be zero, yet the entanglement number for $P_{\psi_{3}}$ and $P_{\psi_{4}}$ are positive.

We now define the entanglement number for a mixed state $\rho$. Suppose $\rho=\sum \lambda_{i} P_{i}, \lambda_{i}>0, \sum \lambda_{i}=1$ is a decomposition of $\rho$ into pure states $P_{i}$, where $P_{i} \neq P_{j}$, $i \neq j$. Let $\mathcal{A}=\left\{P_{i}\right\}$ and define

$$
e_{\mathcal{A}}(\rho)=\sum \lambda_{i} e\left(P_{i}\right)
$$

We define the entanglement number e $(\rho)$ by

$$
e(\rho)=\inf _{\mathcal{A}}\left[e_{\mathcal{A}}(\rho)\right] .
$$

Since a pure state has the decomposition $P=P$, (5) reduces to the usual definition of entanglement number for pure states. We say that the infimum is (5) is attained if there is an $\mathcal{A}$ such that $e(\rho)=e_{\mathcal{A}}(\rho)$. It is an open problem whether the infimum is always attained.

Theorem 10. A state $\rho$ is separable if and only if $e(\rho)$ is attained and $e(\rho)=0$.

Proof. If $\rho$ is separable we have that $\rho=\sum \lambda_{i} P_{i}$ where $P_{i}$ are factorized pure states. Since $e\left(P_{i}\right)=0$ for all $i$, we have that $e_{\mathcal{A}}(\rho)=0$ for $\mathcal{A}=\left\{P_{i}\right\}$. Hence, $e(\rho)=0$. Conversely, suppose $e(\rho)$ is attained at $\mathcal{A}=\left\{P_{i}\right\}$ and $e(\rho)=e_{\mathcal{A}}(\rho)=0$. Since $\rho=\sum \lambda_{i} P_{i}, \lambda_{i}>0, \sum \lambda_{i}=1$ and

$$
\sum \lambda_{i} e\left(P_{i}\right)=e(\rho)=0
$$

we conclude that $e\left(P_{i}\right)=0$ for all $i$. It follows that $P_{i}$ is factorized for all $i$ and hence $\rho$ is separable.

It follows that if $\rho$ is separable, then $e(\rho)=0$ and if $e(\rho)>0$ or is not attained, then $\rho$ is entangled.

\section{References}

[1] P. Busch. The role of entanglement in quantum measurement and information processing. International Journal of Theoretical Physics 2003; 42(5):937-941. doi:10.1023/a:1025462220957.

[2] T. Heinosaari, M. Ziman. The Mathematical Language of Quantum Theory: From Uncertainty to Entanglement. Cambridge University Press, Cambridge, 2012. doi:10.1017/cbo9781139031103.

[3] M. A. Nielsen, I. L. Chuang. Quantum Computation and Quantum Information. Cambridge University Press, Cambridge, 2010. doi:10.1017/ cbo9780511976667.

[4] V. Vedral. Quantum entanglement. Nature Physics 2014; 10(4):256-258. doi : 10.1038/nphys2904

[5] S. Gudder. Quantum entanglement: spooky action at a distance. Quanta 2020; 9(1):1-6. doi: 10.12743/quanta.v9i1.113.

[6] L. Clarisse. On the Schmidt robustness of pure states. Journal of Physics A: Mathematical and General 2006; 39(16):4239-4249. arXiv: quantph/0512012 doi:10.1088/0305-4470/39/16/ Q10.

[7] R. Horodecki, P. Horodecki, M. Horodecki, K. Horodecki. Quantum entanglement. Reviews of Modern Physics 2009; 81(2):865942. arXiv:quant-ph/0702225 doi: 10.1103/RevModPhys.81.865.

[8] M. B. Plenio, S. Virmani. An introduction to entanglement measures. Quantum Information and Computation 2007; 7(1):1-51. arXiv:quant-ph/ Q504163.

[9] M. Steiner. Generalized robustness of entanglement. Physical Review A 2003; 67(5):054305. arXiv:quant-ph/0304009. doi:10.1103/PhysRevA.67.054305.

[10] S. Gudder. A characterization for entangled vectors 2019; arXiv: 1902.08853

[11] S. Gudder. Convex and sequential effect algebras 2018; arXiv: 1802.01265.

[12] S. Gudder. Contexts in quantum measurement theory. Foundations of Physics 2019; 49(6):647-662. arXiv: 1811.10140. doi : $10.1007 / \mathrm{s} 10701-019-00235-5$. 
[13] J. Bae, D. Chruściński, M. Piani. More entanglement implies higher performance in channel discrimination tasks. Physical Review Letters 2019; 122(14):140404. arXiv: 1809.02082. doi:10. 1103/PhysRevLett.122.140404.

[14] V. Vedral, M. B. Plenio. Entanglement measures and purification procedures. Physical Review A 1998; 57(3):1619-1633. arXiv:quant-ph/9707035. doi:10.1103/PhysRevA.57.1619.

[15] G. Vidal, R. Tarrach. Robustness of entan- glement. Physical Review A 1999; 59(1):141155. arXiv:quant-ph/9806094. doi:10.1103/ PhysRevA.59.141.

[16] T.-C. Wei, P. M. Goldbart. Geometric measure of entanglement and applications to bipartite and multipartite quantum states. Physical Review A 2003; 68(4):042307. arXiv: quant-ph/0307219. doi : 10.1103/PhysRevA.68.042307. 